\title{
Nanomembrane: A New MEMS/NEMS Building Block
}

\author{
Jovan Matovic ${ }^{1}$ and Zoran Jakšić 2 \\ ${ }^{1}$ Vienna University of Technology \\ 2Institute of Chemistry, Technology and Metallurgy Belgrade \\ ${ }^{1}$ Austria \\ ${ }^{2}$ Serbia
}

\section{Introduction}

Although MEMS devices are exceptionally diversified, all of them are basically built from a very limited number of constituents manufactured in micrometer-size dimensions: plates, cantilevers, bridges, various channels. It is a combination of these basic building blocks that makes the almost limitless variety of MEMS devices. One may philosophize that at the very foundation of all technologies, MEMS and nanotechnologies included, one encounters atoms and molecules, and the combinations of such basic structural elements can be almost arbitrarily combined to obtain any structure and functionality. In the reality, however, there is a relatively small set of structures behind the existing nanostructures and nanosystems which includes

- Quasi-zero-dimensional structures - nanoparticles (including quantum dots, fullerenes/buckyballs, plasmonic nanoparticles, etc.). This class may be extended to include nanoholes/nanoapertures, nanocavities, etc. which can be seen as the complementary structures to nanoparticles (having a cavity instead of nanoparticle material and a solid instead of the surrounding vacuum or fluid).

- Quasi-1D: various nanorods, nanowires, nanotubes (both carbon and inorganic ones) again including complementary structures.

- Quasi-2D: ultrathin films and membranes.

One may argue that this classification of nanostructural building blocks is actually similar to those already used e.g. in the fields of semiconductor quantum structures where one deals with quantum dots (zero-D), quantum wires (1D) or quantum wells (2D), or in the fields of photonic crystals and metamaterials (1D, 2D and 3D PBG structures), etc.

A plethora of other more complex nanostructures structures may be derived from those already listed and are actually built as their combinations. This includes e.g. quantum rings, various $\mathrm{V}$-shaped nanostructures, nanostrips and ribbons, nanobuds, nanocubes, tripods, spheres, etc., and actually a whole menagerie of the nanotechnological 'zoo'.

It can be seen that plates/membranes appear in both MEMS and nanotechnology building blocks. In the latter, ultrathin membranes represent the basic quasi-2D nanostructure. In MEMS, membranes as structural elements belong to a wider class of microplates.

One possible classification of plates is based solely on their mechanical and geometrical properties. According to the theory of elasticity, plates are divided into three groups, 
depending on their aspect ratio: the thick plates, the diaphragms and the membranes (Timoshenko, 1959). The thick plates balance the external distributed forces (pressure) by a combination of shearing and bending stress. The thick plates find application in MEMS as various supporting structures, rims and other structural elements within the MEMS devices. The diaphragms (thin plates) are somewhat arbitrary defined as plates with an aspect ratio 10 to 100. It is assumed that diaphragms resist the applied pressure solely by bending stress, which is a linear function of the external pressure. Such consideration is of course an approximation, as all three components of stress are simultaneously present in a loaded diaphragm (shear stress, bending stress and membrane stress). The diaphragms are widely used as the sensing element in MEMS pressure sensors.

The last class of plates are membranes, the relatively thinnest plates, with an aspect ratio exceeding 100. This definition is valid both in the macro world as in the MEMS systems. From the point of the theory of elasticity, membranes balance the external pressure exclusively by in-plane membrane stresses, i.e. without a bending rigidity component. A soap bubble or a rubber balloon are typical examples of membrane structures in the macro world. The membrane stress and deflection are highly nonlinear functions of the external load. The application of membranes in mechanical MEMS sensors is limited mostly to the capacitive pressure sensors and microphones where the deflection is small. Membranes find broader application in various bio/chemical sensors, microsleeves and MOEMS devices as e.g. micromirrors.

Regardless of the absolute dimensions or the aspect ratio of the plates used in typical MEMS devices, the basic physical properties of the plate materials remain unaltered when crossing from the macro to the micro world (Bagliom et al, 2007). This is valid for example for Young's modulus, thermal and electrical conductivity and optical properties of MEMS membranes. The values of these material parameters are identical in MEMS and in macro structures. The scaling issues which must be considered in micro-plates with lateral dimensions below $1 \mathrm{~mm}$ are limited to the influence of gravity forces, since the plate mass decreases with the third power of their dimensions.

A new situation, however, arises with a reduction of thickness of the common MEMS membranes under approximately $100 \mathrm{~nm}$. Then the simple MEMS scaling laws are no more fully applicable. In membranes with a thickness of several nanometers and lateral dimensions of the order of a few millimeters, a number of new phenomena appear. For instance, quantum effects become marked in the heat and charge transport, as well as in electromagnetic interactions. Also, the fluid flow models valid for microstructures will not be applicable to the nanoflow featuring the structures with nanometric thickness. A plethora of other novel physical, chemical and mechanical effects appears in these structures, often leading to surprising or even outright counter-intuitive behavior. Such peculiarities classify ultrathin membranes to a separate group of MEMS/NEMS building blocks - the nanomembranes.

Nanomembranes may be defined as freestanding or free-floating artificial or natural structures with a thickness below $100 \mathrm{~nm}$ and a large aspect ratio which may exceed $1.000,000$ (areas of several square centimeters or larger). Their low thickness is very near to the fundamental limit for solids, since typical nanomembranes may be below $5 \mathrm{~nm}$ thick. This approximates 15 atomic layers and makes the nanomembrane structure quasi $2 \mathrm{D}$. In spite of such minute thickness and the extremely large aspect ratio such structures are selfsupported - able to stand free in air or in vacuum, while in the case of free-floating structures they may be even monolayers with a thickness of about $0.3 \mathrm{~nm}$. 
Nanomembranes simultaneously belong to nano-objects (because of their thickness and their low-dimensional physics and chemistry) and to macroscopic objects (because of their large lateral dimensions). A nanomembrane is probably the only nanotechnological object which may be manipulated without special equipment and observed with a naked eye.

Currently the field of nanomembranes is still in its embryonic state regarding the related manufacturing processes, applications, and even the knowledge of their exact composition, structure and behavior. However, this novel paradigm attracts a growing attention of the scientific community and the number of related publication is growing exponentially.

In this chapter we deal with manufactured (artificial, man-made) freestanding nanomembranes and their peculiarities. We review the two major classes of nanomembranes, the inorganic and organic (typically polymeric) ones. We overview the approaches for their fabrication and functionalization. We stress the methods, procedures and structures developed by us. Finally we shortly present some applications of inorganic and organic nanomembranes. Their use as plasmonic structures for chemical, biochemical and biological sensing are presented in a separate chapter of this book.

\section{Terminology and classification}

The fields of science and technology are today more diverse than ever and are further diversifying at an accelerated pace. Many various fields utilize their own terms and idioms and sometimes these are in a discrepancy. This requires that linguistics and semantics follow the explosive growth of science.

The term 'membrane' is a good example, since it depends on the field where it is used. Its general meaning 'any thin, pliable material', and the other, almost equally often met 'a thin, pliable sheet or layer of animal or vegetable tissue serving to line an organ, connect parts, etc.' (Random House, 1992) will not have much meaning in technical sciences.

Membrane has a different meaning in microtechnologies (a relatively thin sheet with a large area, i.e. a large aspect ratio, often made of silicon and possibly having active elements in it), another in biochemistry, third in structural engineering. Previously this usually did not represent a problem, but science is becoming increasingly more multidisciplinary nowadays. Micro- and nanotechnologies include more and more diverse fields and misunderstandings are becoming unavoidable. For instance, if one speaks about a MEMSbased biochemical sensor incorporating a membrane, does this denote a thin, free-standing structure utilized as a (nano)sieve or an active layer containing a MOS transistor?

Nanomembranes may be seen as a special case of general membranes and actually the terminological confusion continues and even deepens here. In a large body of literature a nanomembrane represents a porous membrane with thickness that may be of the order of micrometers, even hundreds of micrometers, but is mesoporous or microporous (van Rijn, 2004). Thus the existence and dimensions of pores are used as the identifier of the whole membrane instead of its thickness and one arrives to a somewhat bizarre situation that the term 'nanomembrane' may denote a structure almost a millimeter thick and with a surface which may exceed square decimeters, only because it has nanometer-sized pores. This goes even further, and according to some sources the 'nanomembrane' may be any membrane if consisting of a nanostructured material (Fissell et al, 2006).

In this Chapter we use the term 'nanomembrane' to denote exclusively a freestanding film with a thickness below $100 \mathrm{~nm}$. The bottom physical limit to the nanomembrane thickness is obviously a molecular or atomic monolayer, i.e. about $0.3 \mathrm{~nm}$. As mentioned previously, the 
lateral dimensions may be large at the same time, of the order of millimeters and even centimeters. It is clear that our definition of nanomembranes does not exclude the existence of nanopores or actually any other kind of functionalization.

Another term relatively often occurring in literature and related with nanomembranes is "ultrathin structure". Such a relative expression is somewhat misleading, and the attribute "ultrathin" is sometimes used for membranes with a thickness of the order of micrometers (Liu et al, 2004).

Some synonyms for the term "nanomembrane" encountered in literature include unbacked films, free standing films, freestanding membranes, ultra-thin (free standing) films, selfsupported films, suspended nanofilms, free-floating films, atomic membranes, monolayer membranes, etc.

When membranes with nanometric thickness are mentioned in literature, very often one encounters structures deposited on some kind of a solid support. A common situation is met in nanofiltration and nanosieves, where the active nanofilm to be used as selective or barrier is deposited over a porous substrate with macroscopic, even mm-order thickness (Pientka et al, 2003). The porous substrate does not hinder the filtrate from passing through, while at the same time it ensures mechanical robustness. Some examples include polymer supports (Sackmann et al, 2000), ceramics (Jayaraman et al, 1995), zeolites (Tavolaro et al, 2007), etc.

This Chapter is dedicated exclusively to nanomembranes as freestanding or free-floating structures which are robust enough to ensure self-support. They may be in contact with gas from one side and with liquid from the other one, or may be completely surrounded by gas or by liquid. For some particular applications, the nanomembrane may be located in vacuum.

As far as their classification is concerned, the basic one would obviously be to artificial (man-made) and biological structures. In this Chapter we deal almost exclusively with the latter.

The artificial nanomembranes can be further divided into two large groups, the inorganic ("hard") ones and the organic ("soft") ones. Basically, one may say that inorganic structures are potentially more robust and are able to withstand more harsh conditions than the organic ones, including high temperatures and aggressive media. However, their simple structure does not leave much space for further functionalization and enhancement.

On the other hand, the organic nanomembranes, which also include the biological ones, are much more sensitive to environmental conditions and are destroyed even at moderately elevated temperatures, are chemically more sensitive and their mechanical robustness is typically poor. At the same time, their "toolbox" contains a virtually infinite number of different materials and the possibilities for functionalization are vast - the diversity of organic life forms and their functions being the prime example.

Obviously, one could also use combinations of both classes to make an infinite number of new composite membranes. It is also possible to use the processes found in biological structures to obtain biomimetic structures enhanced by novel functionalities offered by the nanotechnology.

\section{Artificial nanomembranes and biomimetics}

Biological structures are hierarchically organized. Out of a "toolbox" containing simpler building blocks more complex units are organized, and these units make new sets of building blocks for hierarchically higher toolboxes. 
Nanostructures are near the bottom of this architectural pyramid of life. Biology means nanostructures. Every cell and every part of the cells, from the higher organisms down to bacteria, every virus, every prion are either nanosized structures or their conglomerates. Most of the building blocks of living organisms may be regarded as sophisticated 3D nanosystems.

The nanomembrane as a nanostructural unit is practically unavoidable along the path toward the top of the hierarchical pyramid of life. Typically a living cell includes lipid bilayer membranes incorporating various protein and lipid-based building blocks that enable the functionality of the cell. It divides the cytoplasm of the living cell from its environment and at the same time enables its active interaction with it. The important metabolic processes in each cell proceed through the nanomembrane, but also with its active participation. Throughout the wide variety of life forms, their very existence is enabled through the nanomembranes, which are definitely the most ubiquitous building element of life.

We may look at the living world through the eye of MEMS technologies. There are many reasons that their development nowadays is proceeding in two divergent directions. The first and the oldest approach is to use brute force when manipulating atoms and molecules during the fabrication of a device - the top-down method. A good example is the trend in the development of microprocessors and memory chips. To achieve a larger packaging density one needs cleaner rooms, more precise alignment tools and increasingly accurate process control. In spite of that, today's microprocessors with tens of millions of transistors are unable to emulate the behavior of a simple insect.

It is beyond any doubt that contemporary microtechnologies intensely contributed to an improvement of the overall quality of life - and continue to do so. Never before in any moment of the recorded history it was possible to keep information so safe and to interchange them so quickly. Practically one could not imagine whole fields of human activities without their results. Yet even such a vastly successful approach leaves much to be desired.

Let us consider an example: a swift with a weight of 50 grams and with a tiny brain possesses inertial, topographical, magnetic, solar, lunar and stellar navigation systems. It also has microactuators with sufficiently high power-weight ratio to fly non-stop for thousands of miles; it has built-in temperature controls; growth, reproduction and selfrepair mechanisms; the ability to redesign itself both in the short term (as an adaptation to slightly changing conditions), and in the long term (resulting in another type of machine capable of other skills) (Vincent, 2000). Even our most sophisticated artificial machines are far from such abilities. Yet such an efficient and successful system was realized completely without fascinating facilities and equipment. The key is in the self-organization of the matter according to the nanoscopic blueprint contained in DNA.

Probably the best way to go would be to unite the both approaches, top-down and bottom up, into a single system utilizing the benefits of both. Biomimetics is surely important for this: why not mimic proven solutions from Nature? However, simultaneously one can introduce new ones, which maybe never existed before, into the same structures. Nanotechnologies already offered artificial structures without a known parallel in the real world and at the same time devices and systems closely mimicking the biological ones.

Nanomembranes are an excellent example since they integrate both. If their functionalities were sufficiently good that Nature promoted them into probably the most omnipresent building block of life, why not try and utilize the same already opened path in MEMS/NEMS? 
We have an advantage along the way. We can mimic the nanomembranes offered by the Nature, but at the same time we are free to use the solutions and the functionalities the humanity arrived upon which do not have a parallel in the natural world, especially those introduced by nanoscience and nanotechnologies.

By continuing the development of these fundamental unit blocks of the living world, extending them both into the inorganic and further into organic and imparting them novel functionalities one could hardly expect that many exciting novel findings could not occur. This may well be the missing step to unify both of the existing approaches to the micro- and nanofabrication, thus offering unprecedented functionalities for the welfare of the whole humanity.

\section{General notes on fabrication of artificial nanomembranes}

Various methods for the fabrication of artificial nanomembranes are met within the technological cookbooks of both MEMS technologies and nanotechnologies. Different method tried until now will be presented in more detail further in this Chapter, but in this place we delineate a general procedure used with variations in many different situations. Figure 1 shows its most important technological steps.

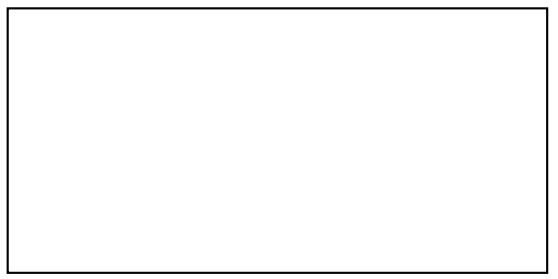

1. Initial structure: sacrificial solid substrate (the alternative is to use liquid as a substrate, in which case step 3 is skipped

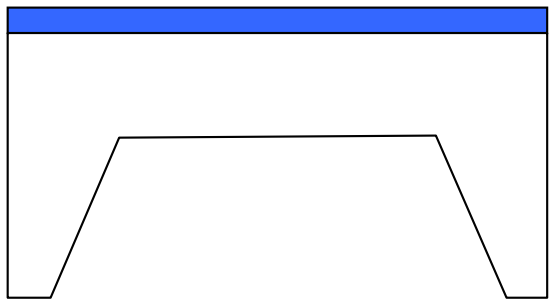

3. Etching/Removal of the sacrificial substrate

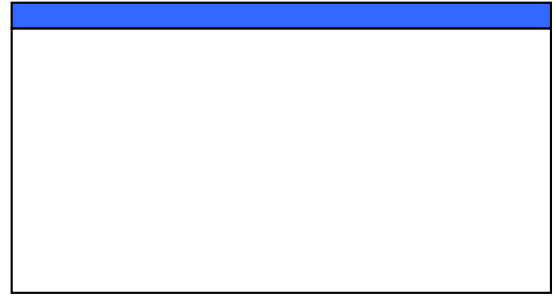

2. Deposition of nanomembrane superstrate (precursor) which may or may not react or mix with the substrate

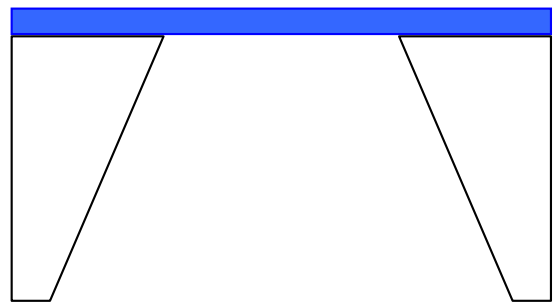

4. Freeing the membrane after the sacrificial structure is fully etched; the rims may remain as support

Fig. 1. Generic technological procedure for the fabrication of nanomembranes utilizing sacrificial support

Most of the approaches start from an initial structure to serve as a support and later to be removed to reveal the nanomembrane (Fig. 1, step 1). This sacrificial structure may be any 
kind of solid, including the traditional materials of MEMS technologies, silicon and silicon dioxide. The choice does not end there, however, since practically any inorganic or organic solids may serve the purpose. Liquid media are also very convenient as supports for the growth of nanomembranes, actually in many situation they are even better choice - not to mention that most biological membranes are grown at a liquid-gas or liquid-liquid interface. A variation of this step is to use a solid or liquid support and apply over it an additional sacrificial layer.

The next step (Fig. 1, step 2) is where the creativity steps in and it differs widely among different methods. The nanometric structure to serve later as a nanomembrane itself is deposited on the support. Practically any material and any deposition method is available to this purpose. One may apply top-down deposition methods or use the bottom up approach. The real knowledge and art are contained in this step. Practically any research team in the field has its own approach and its 'trade secrets' connected with it. Some of the top-down methods used include RF or DC sputtering, electron beam technique, evaporation, electroplating, epitaxy, drop-evaporation method, various versions of chemical or physical vapor deposition, etc. Bottom-up methods include various self-assembly techniques.

The step 2 may also include further processing of the deposited material, to induce further changes in it or to cause its reaction or partial mixing with the substrate. For instance, one may utilize high-temperature annealing to change the crystalline structure/cause reordering of the material, change the structure or remove built-in stresses within the deposited nanolayer, fabricate nanopores, etc.

If a solid substrate is used or an additional sacrificial layer is applied, in step 3 one applies some of the etchants convenient for the particular kind of support material and selective towards the membrane material. For instance, if silicon is used, one may utilize anisotropic etchants like $\mathrm{KOH}$, EDP or TMAH, or isotropic etchants like HF/Nitric/Acetic Acid (HNA). The final step is also very important and may pose large challenges (Fig. 1, step 4). After the etching step is complete and the both sides of the membrane are exposed to the fluid environment, one needs to free the structure from the etchant.

The next two Sections deal with the specific methods to deposit inorganic and organic nanomembranes (step 2 above) and with the properties of thus obtained structures. In both sections the nanomembranes are classified according to their types and composition. For each type the specific fabrication methods and the properties of thus obtained structures are outlined. Such organization of the text was adopted because various types of nanomembranes vastly differ in both fabrication procedures and specific characteristics.

\section{Inorganic nanomembranes}

Although typically with a vastly simpler structure than the organic membranes (and especially the biological ones), the history of the inorganic membranes is, paradoxically, more recent than that of organic membranes. Of course, the first biological nanomembranes appeared simultaneously with the life itself. However, even the artificial organic nanomembranes are at least a century older than the inorganic ones. The existence of inorganic nanomembranes became possible only after the relevant fabrication procedures sufficiently matured to ensure repeatable and reliable production of freestanding nanostructures with extremely high aspect ratios.

In this text we first consider the inorganic structures, because their structure is the simplest one and their properties also shed a light to the function of their more complex organic counterparts. 
There are several main classes of inorganic freestanding structures in dependence on their composition. The simplest one includes pure element nanomembrane and may be divided into three separate subclasses. The first one are pure metal structures; the second one that merits a separate consideration includes predominantly carbon-based nanomembranes, where probably the most important are diamond and diamondoid (diamond-like) ones. The third subclass belongs to elemental semiconductor membranes, where the key position belongs to the silicon structures, a consequence of the dominant position of this material in MEMS and NEMS (more than $90 \%$ of all microsystems are silicon-based).

Another large class includes simple inorganic compounds like oxides, nitrides, carbides and similar mechanically robust materials. Among the oxides, silicon dioxide takes a central position, again because of its dominance in MEMS.

The third important class includes glass and ceramic nanomembranes.

\subsection{Pure metal nanomembranes}

Probably the simplest nanomembranes are those consisting of pure metals. Some of the used materials include chromium, nickel, aluminum, platinum, palladium, silver, gold and similar. One also encounters titanium, tungsten, lead, tin, and copper. A common trait of these materials are that they are structural metals with better or worse mechanical characteristics. Most of them are used in microelectronics and in MEMS, but some of them are known for their use in catalysis. Their chemical inertness is usually rather good.

The history of pure metal nanomembranes can be tracked back to 1931 when Winch manufactured ultrathin freestanding gold films (Winch, 1931). The thickness was $80 \mathrm{~nm}$, a remarkable feat at the time. Manufacturing process was sputtering of gold on the surface of a polished halite mineral (sodium chloride). After gold film deposition, the halite substrate was dissolved in water, leaving the nanomembrane floating free on the water surface.

Not much work on metallic nanomembranes was done in the following decades of the $20^{\text {th }}$ century and most of it was concentrated on very specific applications. We mention here metallic film nanomembranes for the X-ray and the extreme UV spectroscopy, both for atmospheric and space application. The prevalent manufacturing technology was evaporation of metallic layer on a parting substance (Carpenter \& Curico, 1950), (Hunter, 1982). A more advanced technology, evaporation of metals as $\mathrm{Pt}$ and $\mathrm{Cr}$ in the UHV chamber, was used in studies of electron ballistic transport in the ultrathin metallic films (Aristov et al, 1998), (Stepanov et al, 1998), (Glotzer 2004).

The areas of the first metal nanomembranes were typically smaller than $0.1 \mathrm{~mm}^{2}$ and their aspect ratio was below 500. Most of them tended to be brittle and fragile. Metallic nanomembranes are inherently electrically conductive.

Typically the ultrathin pure metal membranes are fabricated utilizing the conventional microsystem technologies, i.e. they used the well known technique of sacrificial supporting structure (Striemer \& Fauchet, 2006), as outlined in Section 4.

According to (Striemer \& Fauchet, 2006), pure metal nanomembranes with surface areas up to about $10 \mathrm{~mm}^{2}$ have been obtained and with aspect ratios up to $430,000: 1$

\subsection{Metal-composite nanomembranes}

As far as the terminology is concerned, the term "composite" within the context of nanomembranes means that their structure consists of a mixture of two or more materials. These may be alloys, single crystals, polycrystals, homogeneous mixture of nanoagglo- 
merates, etc. In this Subsection we stress nanocomposite structures consisting of one or more metals with various additional ingredients, which may include oxides, silicon, etc. Such structures may be uniform or gradient, but they are always homogenized at a level close to the molecular/atomic - or at least at a dimensional level much smaller than the nanomembrane thickness.

In our work we introduced a simple method for the fabrication of nanomembranes with giant aspect ratios exceeding 1.000.000 (Matovic \& Jakšić, 2009). We used only standard procedures of the MEMS technology, although with parameters deviating from the values common in the art. Examples of our metal-composite nanomembranes are shown in Figs. 2 and 3.

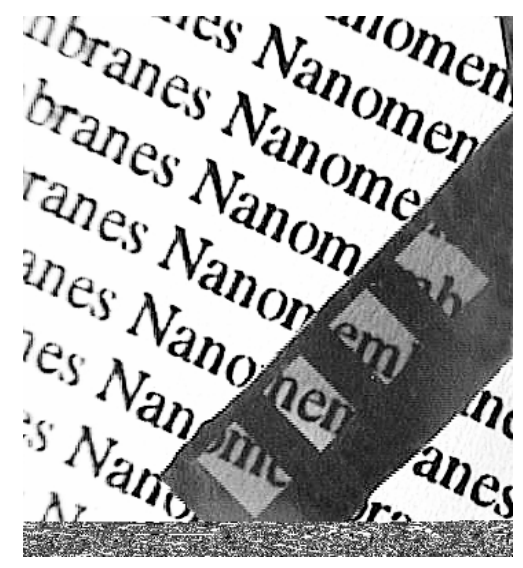

Fig. 2. Photo of an array of metal-composite nanomembranes. Area $1.5 \times 2.5 \mathrm{~mm}$, thickness $7 \mathrm{~nm}$
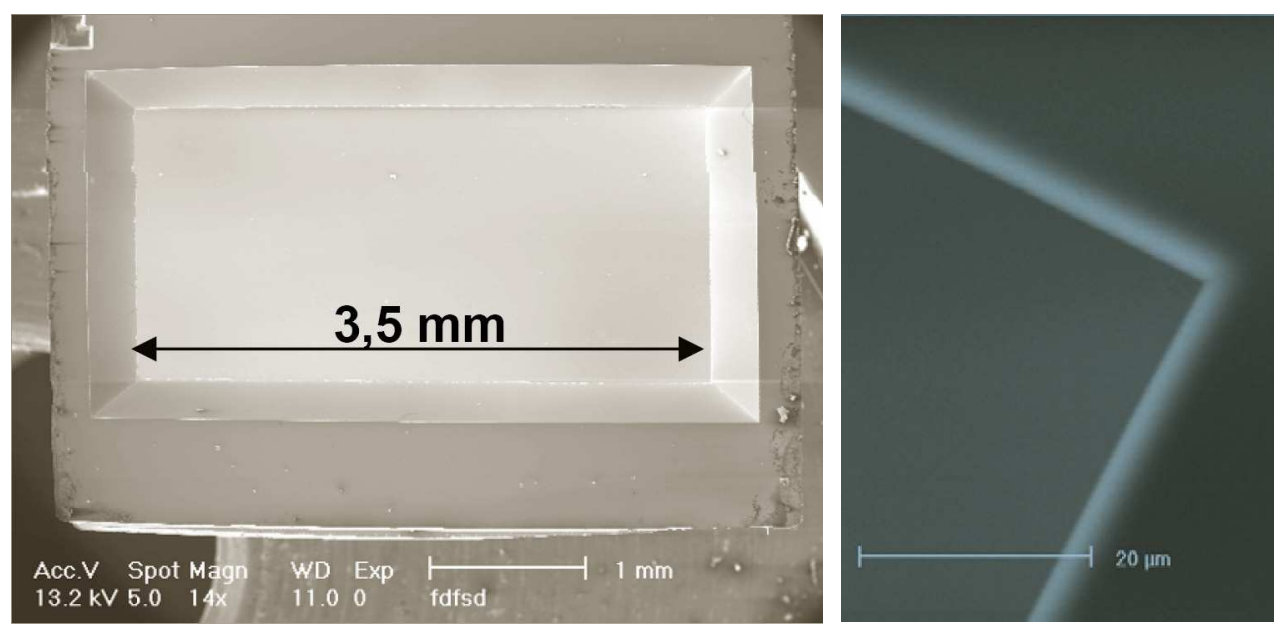

Fig. 3. Giant metal-composite nanomembranes fabricated at TU Vienna. Left: photograph of a $3.5 \mathrm{~mm}$ long nanomembrane, thickness $8 \mathrm{~nm}$. Right: SEM of the edge of the same membrane (Matovic \& Jakšić, 2009) 
Our nanomembranes were made using 50-20 nm thin sputtered films of chromium deposited onto a silicon surface. The sputtering procedure was modified in order to enable formation of a metal-composite complex. A minute and well controlled amount of reactive gas was introduced into the system, which resulted in partial oxidation of the fabricated film. The sputtered chromium atoms were kept at $5 \mathrm{eV}$, allowing a penetration into the sacrificial silicon to a depth of about $0.7 \mathrm{~nm}$. As the subsequent release process we used a back side etch procedure to completely remove sacrificial silicon and free the nanomembrane. The composition of a typical nanomembrane is shown in Fig. 4.

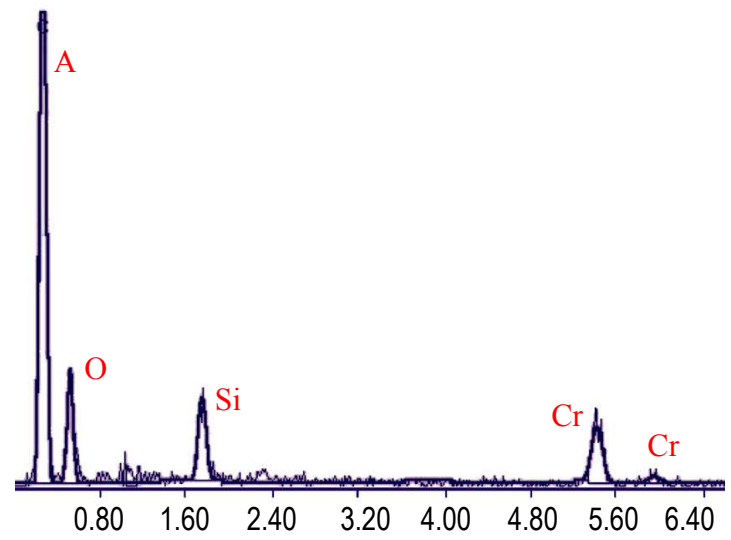

Fig. 4. Energy dispersive X-ray spectrometry analysis of the nanomembrane composition: metal $\sim 52 \%$, oxygen $\sim 28 \%$, silicon $\sim 20 \%$.

The area of metal composite nanomembranes can be increased to several millimeters square, even centimeter square areas. The thickness of our membranes was in the range 5-50 nm, as measured by profilometry after placing the membrane on a polished silicon wafer surface. Until now only the use of oxides with a good adhesion towards the basic metal was confirmed. The application of some complex aggregates, line nanoceramics or clays was not investigated in metallic nanomembranes.

(Vendamme et al, 2006) reported the fabrication of $35 \mathrm{~nm}$ thick nanomembranes based on hybrid interpenetrating networks of organic and inorganic materials. They used simple spin coating to deposit their membranes on the support.

Metal composite nanomembranes are robust on macroscopic level (Fig. 5) and allow aspect ratios in excess of $1,000,000$. They exhibit high flexibility and mechanical strength. They are optically transparent. (Vendamme et al, 2006) reported that their nanomembranes are sufficiently robust to be able to hold amounts of liquid 70,000 times heavier than their own weight, and at the same time sufficiently flexible pass without damages through holes 30,000 times narrower than their size.

For some composite films it was reported that they have a self-healing mechanism able to seal relatively large openings and to restore the structures driven beyond the plastic deformation limit (Jiang et al, 2004). In our own experiments with metal-composite nanomembranes we also observed this effect.

There are important differences in properties between polymer composite and metal composite nanomembranes: 

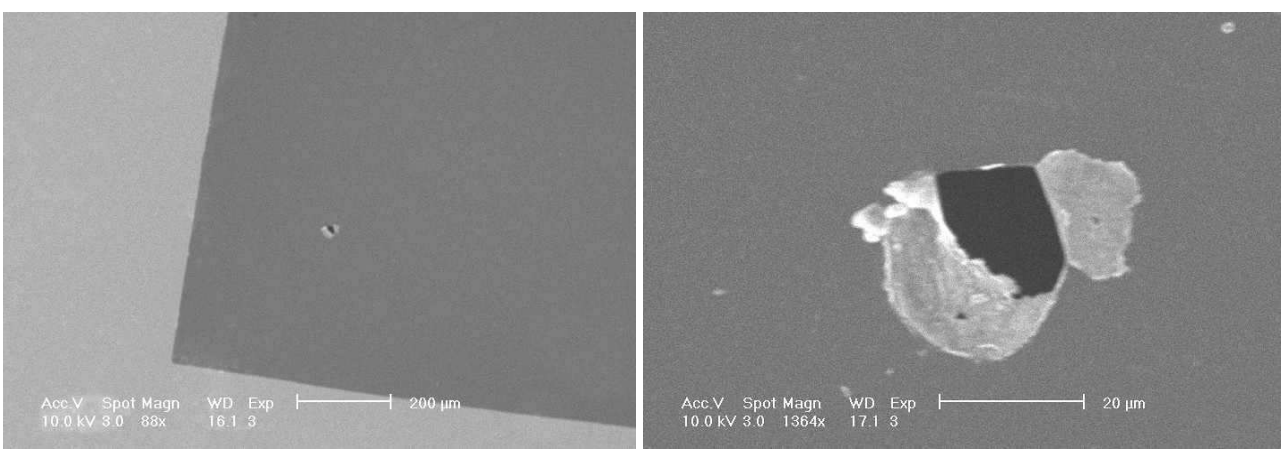

Fig. 5. The example of robustness of a metallic composite NM. SEM picture of a membrane pierced near its edge and a magnified photo of the hole. Even at a large magnification the cross-section of the hole walls is too thin to be observed. The lateral dimensions are $2.5 \times 2.5$ $\mathrm{mm}$, thickness $10 \mathrm{~nm}$.

- Metallic nanomembranes are mechanically more stable than their polymer counterparts. According to preliminary investigations, metallic nanomembranes do not creep under a prolonged stress. Overloaded, they break similar to brittle Si membranes, while under the same conditions polymer nanomembranes creep, see Figure.

- Young's modulus is significantly higher than in polymer composite nanomembranes, although the available data are still not sufficiently reliable

- Metallic nanomembranes are inherently electrically conductive

- Optical transmission is generally lower than in the case of polymer nanomembranes and strongly dependent on thickness. The transmission of nanomembranes 6-7 $\mathrm{nm}$ thick is about $75 \%$, while nanomembranes thicker than $20 \mathrm{~nm}$ are practically opaque and show a mirror-like behavior. However, these properties may be readily engineered to obtain any desired transparency from $0 \%$ to $100 \%$, for instance by utilizing multilayer structures and/or making use of plasmon resonance (Feng et al, 2005 1), (Feng et al, 20052 ).

- The surface roughness of metallic composite nanomembranes is significantly lower than in the case of polymer composite nanomembranes. Already now the mean roughness is below $2 \mathrm{~nm}$, which points out to possible uses for MOEMS devices. Naturally, for some applications it may be desirable to have a higher roughness.

- The robustness of metal-composite nanomembranes is very high. They permit handling with only a modest level of precautions, including the manipulations with wafers supporting nanomembranes using bare fingers (Matovic \& Jakšić, 2009).

\subsection{Carbon, diamond, and diamond-like nanomembranes}

Amorphous, diamond-like carbon films have exhibited excellent material properties such as chemical stability, wear resistance and optical transparency (Petrmichl \& Veerasamy, 2001).. This resulted in their wide use as protective coatings in numerous applications.

Carbon-based diamondoid (diamond-like) nanomembranes were fabricated in Sandia by Friedmann \& Sullivan, 1997). The hydrogenated forms of these films, a-C:H, and specifically the near-frictionless carbon (NFC) films developed at Argonne National Laboratory have exhibited the lowest ever recorded friction coefficient, 0.001 , and ultra-low wear rates of 
10-11-10-10 $\mathrm{mm}^{3} \mathrm{~N}^{-1} \mathrm{~m}^{-1}$ (Robertson, 2002). All of these functionalities are available in freestanding structures (Zhou et al, 2006).

It is even more important that freestanding diamond membranes could be readily combined with any of polymer, metal and other composite nanomembrane materials. Surprisingly, no results of investigations of such structures were published until now, although such synthesis of different technologies is actually very promising and could vastly expand the spectrum of possible applications.

It should be also mentioned that although diamond coatings were investigated as a wide bandgap semiconductor material and generally as microelectronics material, no such investigations were extended to freestanding ultrathin structures, although novel functionalities achieved through the use of quantum effects due to the low dimensionality of these structures could be expected.

\subsection{Elemental semiconductor nanomembranes}

Another class of membranes are those fabricated of single crystalline semiconductors. These may consist of elemental semiconductors, like silicon, compound semiconductors, for instance gallium nitride, silicon carbide, etc., or semiconductor combinations (e.g. latticemismatched strained $\mathrm{Ge}$ on $\mathrm{Si}$, based on the concepts of strain sharing and critical thickness). Such nanomembranes are free-standing, relieved from elastic strain, flexible and dislocation free.

Obviously the central position among the nanomembranes based on elemental semiconductors belongs to silicon. Its technological basis is extremely well developed and mature and the material itself is called "the king of semiconductors". Its mechanical properties make it the material of choice for MEMS systems. Micrometer thick silicon membranes are the basis of many microsystem sensors, but also of a large number of other structures. Nanomembranes represent a natural continuation of the development of such MEMS building blocks to the NEMS area.

Another reason for the fabrication of Si nanomembranes is stretchable electronics (Kim et al, 2008). To this purpose large nanomembranes were made in silicon and applied to different substrates. Buckled, wavy Si nanoribbons and full Si nanomembranes (Choi et al, 2007) with a thickness anywhere between $10 \mathrm{~nm}$ and $100 \mathrm{~nm}$ have been reported. These were fabricated on elastomeric supports of poly(dimethylsiloxane) (PDMS). Full 2D stretchability of such membranes was obtained. Doping, dielectric layers, metallization and other structures were applied to such stretchable/compressible nanomembranes to fabricate MOSFET transistors and $\mathrm{p}$-n junction diodes. Both n-doped and $\mathrm{p}$-doped silicon were used for the fabrication of nanomembranes.

\subsection{Oxide, nitride, carbide nanomembranes}

Various materials belong to this group. Silicon dioxide is most often met. (Striemer, 2006) mentions nanomembranes made from $\mathrm{TiO}_{2}, \mathrm{MgF}_{2}, \mathrm{Ta}_{2} \mathrm{O}_{3}, \mathrm{Y}_{2} \mathrm{O}_{3}, \mathrm{La}_{2} \mathrm{O}_{3}, \mathrm{HfO}_{2}, \mathrm{ZrO}_{2}$. (Branton et al, 2005) describe the fabrication of silica and silicon nitride membranes. Silicon nitride membranes fabricated utilizing the stencil mask technique were reported in (Deshmukh et al, 1999), (Toh et al, 2004). Gallium arsenide nanomembranes were mentioned in (Choi et al, 2007).

It is noticeable that most of the mentioned materials are mechanically robust. They are either dielectrics or wide bandgap semiconductors. Among them, silicon dioxide is relatively least 
robust and it is brittle, however it is extremely well known as a material and its technology is mature, with its roots in microelectronic technologies.

Many (but far from all) of the oxide and generally simple inorganic compound nanomembranes are brittle and fragile, not able to withstand large mechanical forces and with relatively modest aspect ratios.

\subsection{Glass, ceramic nanomembranes}

Glasses and ceramics were also mentioned as materials for experimental nanomembranes. They were used as porous materials, thus enabling the application as nanosieves.

Sodium borosilicate glass was used to fabricate porous membranes with a thickness of about $100 \mathrm{~nm}$ (Enke et al, 2002). To this purpose the initial ultrathin plate of glass was treated by phase-separation and combined acid and alkaline leaching. The pore dimensions in such a membrane were adjusted in the range between 1 and $120 \mathrm{~nm}$.

\section{Organic nanomembranes: macromolecules/ macromolecular composites}

Organic chemistry and biochemistry offer an almost infinite number of macromolecular formulations for nanomembranes, followed with an extraordinarily wide spectrum of chemical, biochemical and physicochemical properties. All macromolecular nanomembranes share some common physical properties. Their mechanical properties are highly sensitive to humidity, temperature and solvents. Further, they have a low Young's modulus, low temperature range and creep under permanent stress. Since nanomembranes thickness is a few dozen of atoms, the instability of their physical properties under the influence of the mentioned agents is more pronounced than in solid bodies with the same composition.

Collodion (pyroxylin) was the first macromolecular nanomembrane (Yamauchi, 2000). It was discovered in 19th Century. Collodions were fabricated by solving the nitrocellulose in acetone. The solution was poured to the water surface and after acetone evaporated, it left a thin nitrocellulose film, floating on the water surface. The film was released using a wire loop. Thus a free-standing nanomembrane was obtained, and it was relatively stable and durable. Collodion is practically forgotten nowadays, but the described method of nanomembrane fabrication is today among the mainstream ones: one of the many examples that today's technologies often rest on a knowledge accumulated for a long period of time.

Some organic nanomembranes are based on liquid crystalline films (Sonin, 1999), but they are very unstable. Further, every year hundreds of papers are published on LangmuirBlodgett (LB) method (Peterson, 1990), which allows one to construct amphiphile multilayers with a thickness ranging from 5 to $500 \mathrm{~nm}$. A Langmuir-Blodgett film is formed by one or more organic monolayers on a liquid surface and is often transferred to a solid surface through its immersion into or emersion from the liquid. Other applicable methods are based on thiol or silane compounds, spin coating and thermal deposition of macromolecules onto a substrate. Unfortunately, these methods do not allow one to control the molecular order in the films.

Finally, there is a more recent method for film self-assembly that makes use of the alternate adsorption of oppositely charged macromolecules (polymers, nanoparticles, proteins) - the Layer-by-Layer technique (LbL) (Lvov et al, 1995). The assembly of alternating layers of oppositely charged long chained molecules is a simple process, closely mimicking the natural self-organized structures. The process provides the means to form 5-500 $\mathrm{nm}$ thick 
films with monolayers of various substances growing in a pre-set sequence on a substrates at a growth step of about $1 \mathrm{~nm}$ per cycle. These nanomembranes have a lower molecular order than the LB films, but the advantages are high strength and easy preparation. This technique has been called in some places "molecular beaker epitaxy," meaning by this that with simple instruments (exploiting the materials self-assembly tendency) one can produce molecularly organized films similar to the ones obtained with highly sophisticated and expensive molecular beam epitaxy technology used in top-down approach.

Briefly, the LbL process proceeds as follows. A substrate is immersed into a dilute solution of a cationic polyelectrolyte, optimized for the adsorption of a single monolayer (ca $1 \mathrm{~nm}$ thick), rinsed and dried. The next step is the immersion of the polycation-covered substrate into a dilute dispersion of polyanions or negatively charged nanoparticles, also optimized for the adsorption of a monolayer, and then rinsed and dried. These operations complete one cycle of the self-assembly of a polyelectrolyte monolayer onto the substrate. (Fig. 6).

The subsequent sandwich units are analogously self-assembled. Linear polycation/polyanion multilayers can be assembled by similar means. Different molecules, proteins, enzymes, antigens and so on may be assembled in a pre-planned order in a single film.
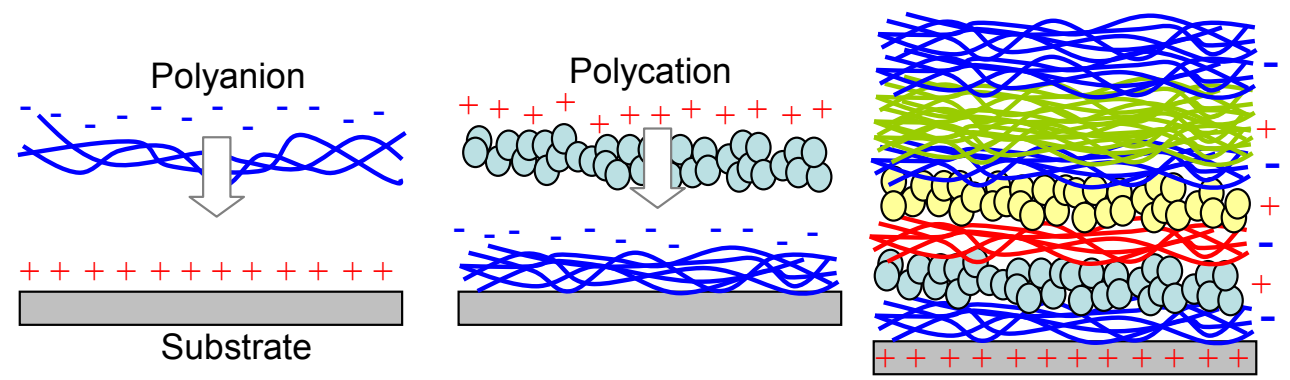

Fig. 6. Illustration of the layer-by-layer nanomembrane assembly technique. Left: assembly of the first layer; middle: assembly of the second layer; right: final structure.

\section{Basic methods of nanomembrane functionalization}

Nanomembrane functionalization is the essential step towards fundamental extension of their application. This includes an imparting additional desirable mechanical, electronic, chemical, biological, optical, magnetic, etc. nanomembrane properties. Thus it represents an essential factor in extending their practical applicability.

\subsection{Nanomembrane functionalization by active fillers}

An obvious method to enhance the performance of nanomembrane is to include fillers into their matrix, for instance nanoparticles with desired properties, and thus to create an enhanced nanocomposite (Zhang \& Ali, 2007). In the current implementations the presence of fine fillers or clusters in the nanomembrane matrix mostly enriches the durability and overall mechanical properties of the nanomembrane. Such nanofillers are the passive (inactive) ones.

Inactive fillers can be replaced by nanoparticles already possessing an additional functionality, for instance catalytic $(\mathrm{Pt}, \mathrm{Ag}, \mathrm{Rh})$, photocatalytic $\left(\mathrm{TiO}_{2}\right)$, light-emitting $(\mathrm{SiC}$, 
ZnSe), soft and hard magnetic nanoparticles, electrets or piezoelectric ceramics, chemically active substances, etc. The fillers may consist of nanoparticles or structures with inherent multifunctionality, like fullerenes of nanotubes. Fig. 7 shows some possible nanofillers.

The introduction of active nanoparticle fillers should be performed without compromising the existing mechanical properties of the nanomembranes.

Nanoparticle incorporation may be done during the production of the membranes themselves (using any of the various methods like incorporation by melt compounding, incorporation during polymerization step, blending and hot (dry/wet) isostatic pressing, plasma spraying techniques and co-evaporation / codeposition, etc).

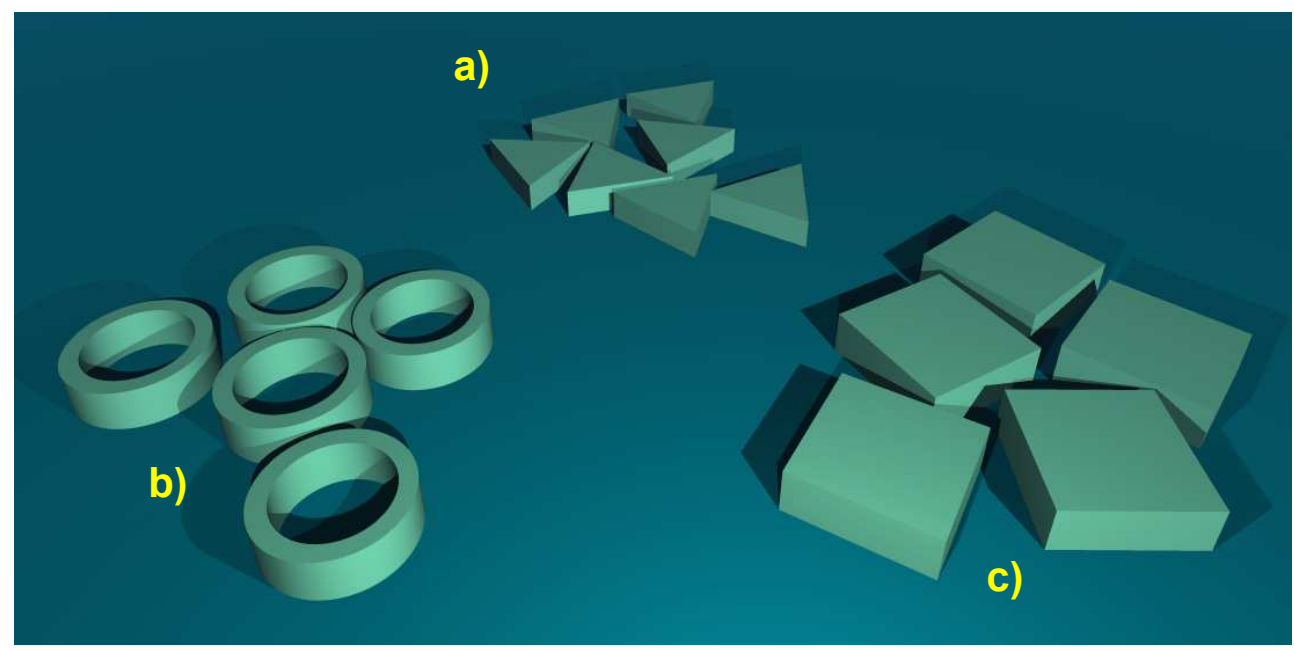

Fig. 7. Various forms of nano-fillers. a) triangular nanoprisms (may be fabricated in gold); b) nanorings (zinc oxide); c) nano-cubes (silver). The nano-building blocks are not to scale.

\subsection{Lamination}

The simplest way to introduce multiple functionalities to nanomembranes is to fabricate sandwich structures, i.e. to perform lamination. The fabricated superstructures may consist of two (the simplest case) or more strata. Each stratum introduces its own properties to the nanocomposite. For instance, one may be conductive; another may ensure mechanical strength and robustness. One may introduce a layer to serve as a plasmonic waveguide and another may be a ligand layer to attract a specific chemical or biochemical only.

Fig. 8 shows several possible configurations for nanomembrane functionalization through lamination. Obviously, the number of possible combinations of properties and functions is virtually limitless, and the basic limitation stems from the adhesion and reactions between different strata.

An important kind of functionalization through lamination is passivation treatment, necessary for mechanically sensitive or unstable surfaces, for those reactive, oxidizable, etc. Such treatment includes diamond-like materials, polymers, metals, semiconductors and their combinations. Chemical modification may be done by surfactants. 

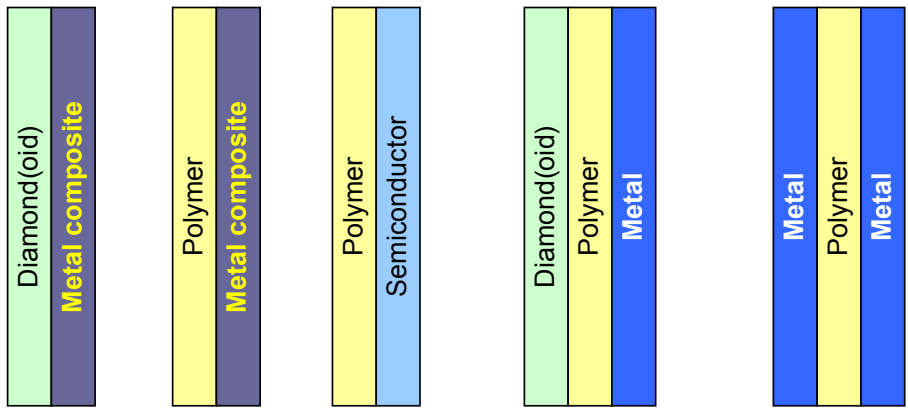

Fig. 8. Several possible basic kinds of nanomembrane functionalization through lamination.

\subsection{Structural modification}

Another large group of functionalization methods is based on patterning of nanomembrane structures. For instance, one may form 2D arrays of nanoholes/nanoapertures with controlled geometry in the nanomembranes. Fabrication of 2D nanohole (nanopore) arrays also belongs to nanopatterning and brings with itself another group of functionalities (Fig. 9). Nanopatterning of membranes may be done for instance by microcontact printing, using dry lift-off approaches and many other approaches (Hannink, 2006). Due to the extremely small thickness of nanomembranes the ratio between the pore width and the nanomembrane thickness remains small. Due to this, it is much easer to maintain the control of the pore dimensions during micro/nanofabrication, since the aspect ratio (diameter to width) of the pores themselves is low.

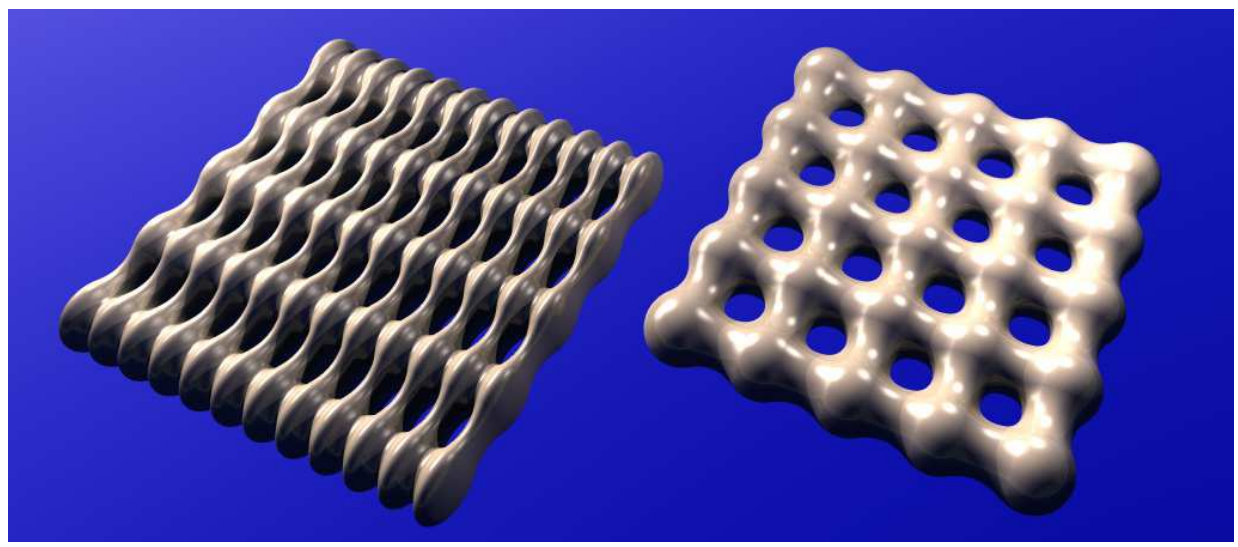

Fig. 9. Structural modification of nanomembranes through formation of 2D nanoaperture patterns; left: elliptical openings; right: circular openings.

A method of forming pores in freestanding silicon nanomembranes was described by (Striemer et al, 2007), Fig. 10. After depositing a silica-silicon-silica sandwich onto a Si sacrificial substrate one anneals the Si nanomembrane precursor and as a result pores are formed. The higher the annealing temperature, the larger is the pore size. It can be seen that the procedure is a variation of the generic sacrificial process for nanomembrane fabrication, as outlined in Section 4. 


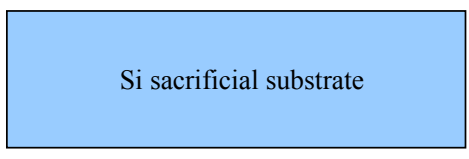

a)

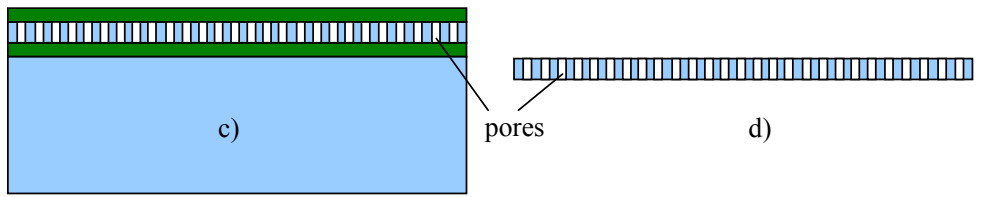

b)

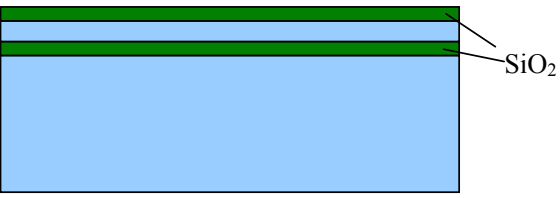

Fig. 10. Fabrication of pores in Si nanomembrane. a) Sacrificial silicon substrate; b) layers of silicon dioxide, silicon and again silicon dioxide are deposited; c) after annealing, pores are formed in silicon; d) sacrificial Si is etched away.

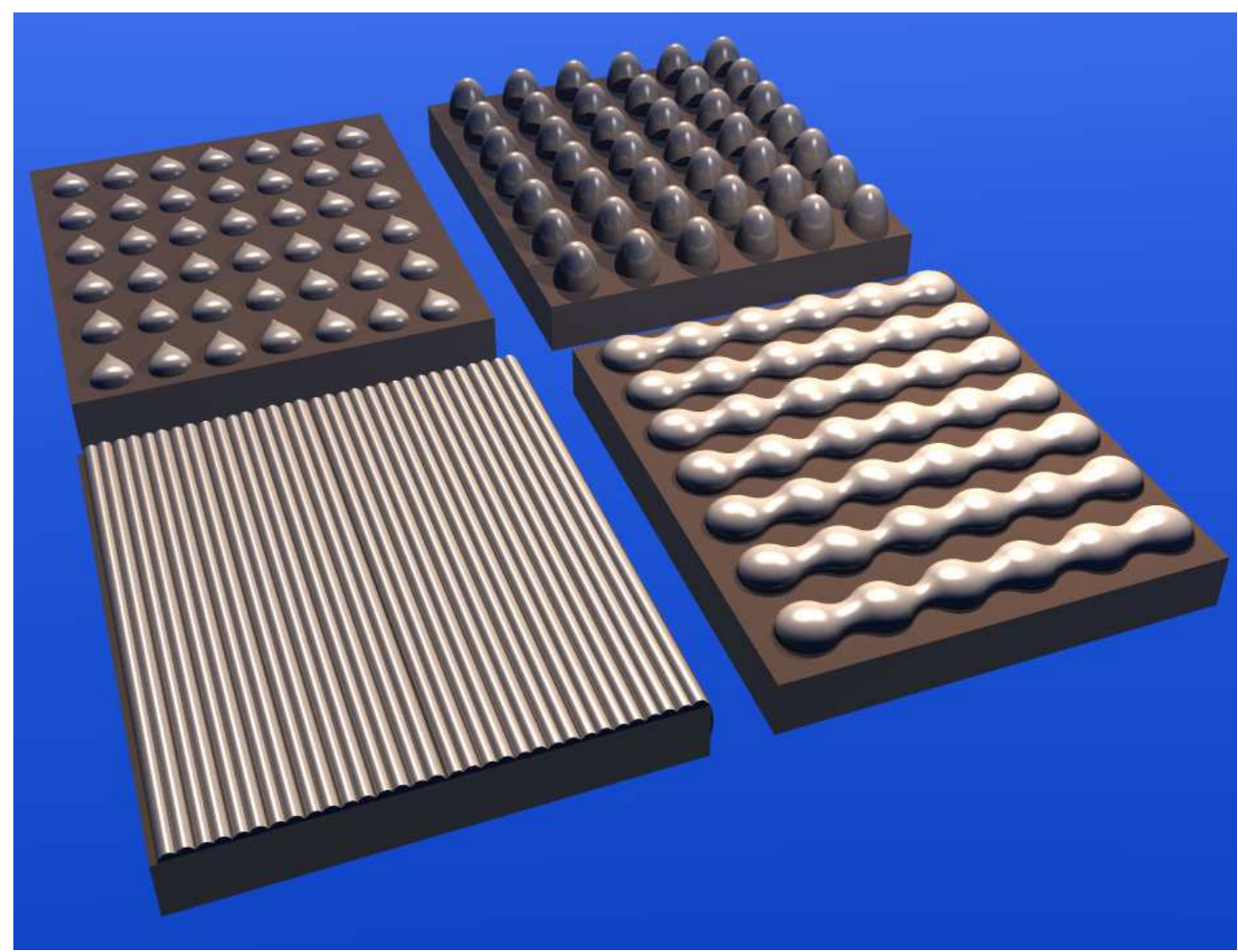

Fig. 11. Nanomembrane modification through various forms of surface modification.

\subsection{Surface modification}

An approach which may be regarded as a subgroup of the structural modification is the modification of the nanomembrane surface. However, this is a very rich and diverse group in itself and includes a number of subclasses. Some of those include 
- nanopatterning/deposition of various planar and 3D structures directly to the nanomembrane surface. These patterns may modify the electromagnetic or optical behavior of the surface, enhance or suppress local wettability, improve attraction or repellence toward biological/living agents, etc.

- chemical, biochemical or biological activation through deposition of e.g. ligands, catalysts, etc.

- Passivation surface treatment, necessary for mechanically or chemically unstable nanomembranes (e.g. oxidizable, reactive, etc.)

Some surface modifications obtainable through the application of nanolithography are shown in Fig. 11. The alterations may be done in the nanomembrane material itself or through the deposition of an additional pattern which may assume the form of various pillars, stripes, etc.

\section{Some basic applications of nanomembranes}

Since nanomembranes are a novel concept which extends the range of MEMS \& NEMS building blocks and practically introduces a new one, this means that whole branches of science and technology can be re-read and re-created through it, which obviously has a potential to create an enormous number of novel applications. In this Chapter we only give a brief summary of some of the potential applications. Practically it may be said that the nanomembranes are applicable everywhere where microfabrication, MEMS/NEMS and nanotechnologies are applicable. One of the reasons for this is their unique property to combine nanometric dimension in one direction with $\mathrm{mm}-$, even $\mathrm{cm}$ in other dimensions, thus offering nanotechnological functionality with an ease of manipulation, use and characterization.

The potential applications of nanomembranes can be roughly divided into two groups:

1. Quantitative and qualitative improvements of the known applications of conventional membranes.

New physical properties brought by the low-dimensionality of nanometric order are sure to result in improvements of the existing or already proposed functionalities

2. Qualitatively new functionalities brought by low-dimensional physics of nanomembranes.

Nanomembranes possess a plethora of novel properties which are yet to be investigated and their applications found. Already those investigated and proposed until now seem extremely promising. For instance, nanomembranes represent a generalization not only of conventional membranes/diaphragms in MEMS/NEMS, but also of microbridges and nanocantilevers (obtainable by forming the nanomembrane). Novel applications may be expected from their use in e.g. subwavelength optics, electromagnetics; quantum behavior resulting from their extreme low-dimensionality also offers new functionalities, etc. One may claim that whole fields of technology and application should be reviewed through the possibilities offered by the existence of membranes an order of magnitude thinner than those conventionally used.

Among the obvious properties of nanomembranes is a vastly improved ratio of the effective area to the total volume of the structure compared to thicker structures. In Fig. 12 one may see two membranes contained in an identical volume, but with different thickness. Although the ratio of their thickness is about an order of magnitude only, one can see that the effective area is much larger for the thinner membrane. In reality the ratios are much larger, and may well be 3-6 orders of magnitude. Due to the simple scaling issues (surface 
decreasing with the second power, volume with the third power of the geometrical dimensions) it is obvious that the surface to volume ratio may be very large. This is of immense importance for various catalytic/enzymatic processes. One may expect higher conversion efficiencies and simultaneously a significant reduction of the necessary amount of expensive metals (e.g. platinum, palladium) in catalytic systems like microreactors, microconvertors, etc.

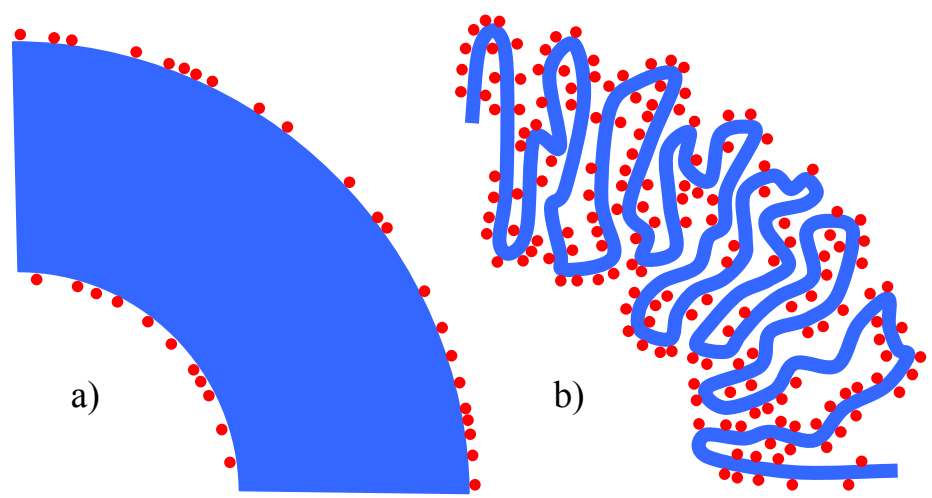

Fig. 12. Comparison of effective areas for adsorption of two membranes with different thickness, but contained in an identical volume. The number of adsorbed particles, being proportional to the area, is obviously larger in (b).

Among devices that could make use of the improved catalytic properties of nanomembranes are micro-batteries and battery arrays, various kinds of fuel generators and fuel converters, fuel purification systems (ranging from those for e.g. gasoline desulphurization to inexpensive and low-scale nuclear fuel purification). Especially, the nanomembranes may be used as a viable alternative to the conventional Nafion ${ }^{\circledR}$-based membranes (Hamrock \& Yandrasits, 2006) in proton-exchange fuel cells to improve their efficiency.

Another large field of nanomembrane application is for nanosieves and nanofilters. In nanomembranes the aspect ratio between the pore radius and the overall structure thickness is much smaller than in conventional porous membranes, which are typically $1000-10,000$ times thicker than the particles to be separated (Striemer et al, 2007). A smaller aspect ratio means that the filtered particle has to pass a much shorter path through the filtering channel and consequently the transport rates/throughput will be larger. Also, in structures with a smaller aspect ratio it is also much easier to control and tune the cross section of the nanoaperture. This may result in an improved selectivity of the nanosieve and avoidance of the loss of filtrate within the membrane itself. In nanomembranes it is also easier to control the distribution of electrical charges within the channel, which will also improve filtering selectivity, this time by influencing the transport of polar molecules.

At the same time, the possibility to use novel strategies for nanomembrane fabrication enables to make them mechanically much more robust than the conventional structures. As an example, $\mathrm{SiN}_{\mathrm{x}}$ nanomembranes incorporating nanohole arrays and intended for the use as filters and bioseparators) (Tong et al, 2004) about 10-30 nm thick are able to withstand single-sided overpressures of about 10 bar.

Nanosieves may be utilized for diverse applications, ranging from fluid-separation and gasseparation units and various size-exclusion-based separations and extractions to molecular 
and microbial sieves for separation and purification of organic agents (for instance, absolute sterile filtration of bacteria \& viruses). Among the important applications are DNA and microorganism separation, different proteomics devices and the fields of use include nanomedicine, genetic treatment and analysis, microbiology, biochemistry, bioseparation, but also environmental sensing and chemical/biochemical sensing generally (templates for nanosensors), homeland security, pathogen recognition, and also nanofabrication (e.g. masks for stencils) etc.

An important field of the application of nanomembranes is fuel purification and separation, including hydrogen recovery from gas mixtures and hydrocarbon fractionation. Another one is water desalination, detoxification and purification. The reverse osmosis by the use of nanomembranes was described in (Murad \& Nitsche, 2004). Ion separation by macromolecular nanomembranes was elaborated in (Toutianoush \& Tieke, 2001). An additional feature for filtering applications may be expected from a combination of nanomembranes with pores and the application of electrostatic potential, since the resulting buildup of the electric field enhances the selective rejection of ions (Schaldach et al, 2004). Further, in biological/non-biological interfaces a nanomembrane may serve as an "ideal" biomimetic (enhanced or not) biological/nonbiological interface, convenient for chemical functionalization, patterning at the micro and nanometer scale, but also enabling engineering at macroscale (Watanabe \& Ishihara, 2008). It may serve as a substrate for immobilization of proteins, antibodies, enzymes, cells through pattering a 2D arrays of anchoring units (DNA footprinting platform).

\subsection{Main fields of nanomembrane application}

In the further text, we present the main fields of nanomembrane application. For the convenience, we divided possible applications of nanomembranes into several main fields of interest and quoted only a few examples for each.

\section{Energy production and conversion}

- Fuel cells; other micro-power sources and microbattery arrays; nuclear fuel production/purification; selective removal, separation, extraction of hydrogen; gasoline desulphurization; petrochemistry; hydrocarbon fractionation; fuel production (environment-friendly, microreactor-based, biomimetic); fuel purification/separation (environment-friendly, microreactor-based).

\section{Biomedicine}

- Drug delivery systems; cancer treatment (selective drug delivery/selective enzyme removal); hemodialysis; blood treatment/filtration; medical sensors incl. implantable devices; diagnostic instruments; healthcare systems; immune system investigation.

\section{Bioengineering}

- $\quad$ DNA analysis/separation; gene analysis/research, genomics; cell biology and biotechnology; nanosieves; protein biomarker detection; virus and prion analysis; fabrication of diverse biomimetic nanostructures; biochemical sensing.

\section{Chemical Engineering}

- Food and beverage processing; seawater desalination; multicomponent gas mixtures separation; gas dehydration; other chemicals separation; reverse osmosis filtration; 
chemicals analysis; microfiltration; ultrafiltration; nanofiltration; ultrapure chemicals production (environment-friendly, microreactor-based); $\mu$-separators; atomization; emulsification.

\section{Environmental Protection}

- Recover volatile organic compounds from airstreams; wastewater treatment; air pollution control; recovery of valuable chemicals; water recycling; potable water production/purification; chemical, biochemical and biological sensors.

\section{Toxicology, forensics and homeland defense}

- Recognition of harmful inorganic, organic and biological agents.

\section{MEMS/NEMS}

Novel MEMS/NEMS devices (expanding the limits of all MEMS/NEMS building blocks to nanometer thickness and offering a whole new class of free-standing structures); various physical and chemical sensors for process industry, automotive industry, airspace, etc. including highly sensitive pressure and acoustic microsensors; nanosieves; self-recoverable micro and nanostructures; very high frequency microoscillators and microresonators; catalytic membrane microreactors; high temperature microreactors; "Lab on a chip"; molecular transport and sorting; nano printing and etching; stenciling using nanosieves.

\section{Semiconductors}

- e-projection lithography; subwavelength lithography based on "superlensing"; purification of microelectronics grade rinse water; interconnects and conductors; "superconductive" circuit components; flexible electronics.

\section{Optics \& Electromagnetics}

- Free-standing integrated optics; thermal detectors; radiation and particle detectors; subwavelength optics; micro- and nano subwavelength waveguides; delay lines; plasmonic sensors; nanoplasmonic devices; nanophotonic structures; electromagnetic and optical metamaterials; cloaking devices; electric screening; supercapacitors; displays; projectors; light sources; nonlinear optical elements with second harmonic generation; extreme UV and X-ray applications.

\section{Conclusion}

Since nanomembranes are a novel concept which extends the range of MEMS \& NEMS building blocks and practically introduces a new one, this means that whole branches of science and technology can be re-read and re-created through it, which may create an enormous number of novel applications. Nanomembranes need to be incorporated into coherent and ambitious programs of nanotechnology research, with aggressive funding and awareness-increasing campaigns. A care should be taken at that both about the fundamental and the applied aspects of research, since the recent developments clearly indicate that the field may have many promises and even surprises in stock.

A development of a novel technology or concept very rarely follows a smooth and gradual trend. Much more often one encounters an abrupt surge in development after the necessary conditions are met, not only scientific and technological, but also social and economic. In our opinion such is the situation with nanomembranes at the beginning of the 21st Century. 


\section{Acknowledgments}

This project is supported by the Austrian FFG Fund under the program Climate and Energy Fund and the under the program "ENERGY FOR THE FUTURE" ("ENERGIE DER ZUKUNFT") within the project NanomembPEMFC "Advanced Generation of Proton Exchange Fuel Cells utilizing nanomembrane assembly" and by the Serbian Ministry of Science and technology within the project 11027 "Microsystem and Nanosystem Technologies and Devices".

\section{References}

Aristov V.V., Kazmiruk V.V., Kudryashov V.A., Levashov V.I., Redkin S.I. Hagen C.W., and Kruit, P. (1998), Microfabrication of ultrathin free-standing platinum foils, Surface Science Vols. 402-404, pp. 337-340.

Bagliom, S; Castorina, S; and Savalli, S. (2007), Scaling Issues and Design of MEMS, John Wiley \& Sons Ltd, ISBN 978-0-470-01699-2

Branton, D.; Gordon, R. G.; Chen, P.; Mitsui, T.; Farmer, D. B. and Golovchenko, J. (2005), Analysis of molecules by translocation through a coated aperture, WIPO Patent WO/2005/061373.

Carpenter F.E., Curico J.A, (1950), Preparation of Unbacked Metallic Films, Review of Scientific Instruments, Vol. 21, pp. 675- 676

Choi, W. M.; Song, J.; Khang, D.-Y.; Jiang, H.; Huang, Y. Y. and Rogers, J. A. (2007), Biaxially Stretchable "Wavy" Silicon Nanomembranes, Nano Letters, Vol. 7, No. 6, pp. 16551663.

Deshmukh, M. M.; Ralph, D. C.; Thomas, M. and Silcox, J. (1999), Nanofabrication Using a Stencil Mask, Applied Physics Letters Vol. 75, pp. 1631-1633.

Enke, D.; Friedel, F.; Janowski, F.; Hahn, T.; Gille, W.; Müller, R. and Kaden, H. (2002), Ultrathin porous glass membranes with controlled texture properties, Studies in Surface Science and Catalysis, Vol. 144, pp. 347-354.

Feng, S.; Elson, J. M.; Overfelt, P. L.; (2005) (1) Optical properties of multilayer metaldielectric nanofilms with all-evanescent modes," Optics Express Vol. 13, p. 4113-4124.

Feng, S.; Elson, J. M.; Overfelt, P. L.; (2005) (2) Transparent photonic band in metallodielectric nanostructures," Physical Review B Vol. 72, 085117

Fissell, W. H., IV; Humes, D. H.; Roy, S.; and Fleischman, A. (2006) Ultrafiltration membrane, device, bioartificial organ, and methods, United States Patent 20060213836

Friedmann, T.A. and Sullivan, J.P., (1997) Thick stress-free amorphous-tetrahedral carbon films with hardness near that of diamond, Applied Physics Letters, Vol. 71, No. 26, pp. 3820-3822.

Glotzer, S. C.; Solomon, M. J., and Kotov, N. A. (2004), Self-assembly: From nanoscale to microscale colloids, AIChE Journal, Vol. 50, No. 12, pp. 2978 - 2985.

Hamrock, S. J. and Yandrasits, M. A. (2006), Review: Proton Exchange Membranes for Fuel Cell Applications, Journal of Macromolecular Science, Part C: Polymer Reviews, Vol. 46, pp. 219-244

Hannink, R. H. J. (2006), Nanostructure control of materials, CRC, Woodhead Publishing Limited 
Hunter W. R. (1982), Measurement of optical properties of materials in the vacuum ultraviolet spectral region, Applied Optics 2103, Vol. 21, No. 12, pp. 2103-2114.

Jayaraman, V.; Lin, Y. S.; Pakala, M. and Lin, R. Y. (1995), Fabrication of ultrathin metallic membranes on ceramic supports by sputter deposition", Journal of Membrane Science Vol. 99, No. 1, pp. 89-100.

Jiang, C.; Markutsya, S. \& Tsukruk, V. V. (2004). Compliant, robust, and truly nanoscale free-standing multilayer films fabricated using spin-assisted layer-by-layer assembly. Advanced Materials 16, 157-161., ISSN: 0935-9648.

Kim, D.-H.; Ahn, J.-H.; Choi, W. M.; Kim, H.-S.; Kim, T.-H.; Song, J.; Huang, Y. Y.; Liu, Z.; Lu, C. and Rogers, J. A. (2008), Stretchable and Foldable Silicon Integrated Circuits, Science Vol. 320, pp. 507-511.

Liu, L.; Chakma, A.; and Feng, X. (2004), A novel method of preparing ultrathin poly(ether block amide) membranes, Journal of Membrane Science, Vol. 235, Nos. 1-2, pp. 43-52.

Lvov, Y.; Ichinose, I. and Kunitake, T. (1995), Assembly of Multicomponent Protein Films by Means of Electrostatic Layer-by-Layer Adsorption, Journal of the American Chemical Society, Vol. 117, pp. 6117-6122.

Murad, S. and Nitsche, L. C. (2004), The effect of thickness, pore size and structure of a nanomembrane on the flux and selectivity in reverse osmosis separations: a molecular dynamics study, Chemical Physics Letters, Vol. 397, Nos. 1-3, pp. 211-215.

Peterson, I.R. (1990), Langmuir Blodgett Films , Journal of Physics D, Vol. 23, No. 4, pp. 37995.

Petrmichl, R. H. \& Veerasamy, V. S. (2001), Hydrophobic coating with DLC \& FAS on substrate, United States Patent 6312808.

Pientka, Z.; Brožová, L.; Bleha, M.; Puri, P. (2003), Preparation and characterization of ultrathin polymeric films, Journal of Membrane Science, Vol. 214, No. 1, pp. 157-161.

Random House Webster's Unabridged Dictionary, Random House Reference (2003).

Robertson J. (2002), Diamond-like amorphous carbon, Material Science and Engineering R Vol. 37, pp. 129-281.

Sackmann, E. and Tanaka, M. (2000), Supported membranes on soft polymer cushions: fabrication, characterization and applications, Trends in Biotechnology, Vol. 18, No. 2, pp. 58-64.

Schaldach, C. M.; Bourcier, W. L.; Paul, P. H. and Wilson, W. D. (2004), Electrostatic potentials and fields in the vicinity of engineered nanostructures, Journal of Colloid and Interface Science, 275 (2), pp. 601-611.

Sonin A. A. (1999), Freely Suspended Liquid Crystalline Films, John Wiley \& Sons, ISBN-13: 978-0471971559

Stepanov, I.S.;, van Aken, R.H.; Zuiddam, M.R., and Hagen, C.W. ; Fabrication of Ultra-Thin Free-Standing Chromium Foils Supported by a $\mathrm{Si}_{3} \mathrm{~N}_{4}$, Membrane-Structure with Search Pattern, Microelectronic Engineering, Vol. 46, No 1-4, pp. 435-438, ISSN 01679317.

Striemer, C. C., and Fauchet, P. M., (2006), Ultrathin nanoscale membranes, methods of making, and uses thereof, United States Patent 20060243655

Striemer, C. C.; Gaborski, T. R.; McGrath, J. L.; Fauchet, P. M. (2007), Charge- and size- based separation of macromolecules using ultrathin silicon membranes, Nature Vol. 445, pp. 749-753. 
Tavolaro, A.; Tavolaro, P., and Drioli, E. (2007), Zeolite inorganic supports for BSA immobilization: Comparative study of several zeolite crystals and composite membranes, Colloids and Surfaces B: Biointerfaces, Vol. 55, No. 1, pp. 67-76.

Timoshenko, S. and Woinowsky-Krieger S. (1959), Theory of Plates and Shells, McGraw-Hill Co. 2 edition, ISBN-13: 978-0070647794

Toh, C. S.; Kayes, B. M.; Nemanick, E. J. and Lewis, N. S. (2004), Fabrication of Free-Standing Nanoscale Alumina Membranes with Controllable Pore Aspect Ratios, Nano Letters Vol. 4, pp. 767-770.

Tong, H. D.; Jansen, H. V.; Gadgil, V. J.; Bostan, C. G.; Berenschot, E.; van Rijn, C. J. M. and Elwenspoek, M. (2004), Silicon nitride nanosieve membrane. Nano letters, Vol. 4, No. 2, pp. 283-287.

Toutianoush, A. and Tieke, B. (2001), Ultrathin self-assembled polyvinylamine /polyvinylsulfate membranes for separation of ions", Studies in Interface Science, Vol. 11, pp. 415-425.

van Rijn, CJM (2004), Nano And Micro Engineered Membrane Technology, Elsevier, 2004, ISBN13: 978-0-444-51489-9

Vendamme, R.; Onoue, S. Y.; Nakao, A.; Kunitake, T., (2006), Robust free-standing nanomembranes of organic/inorganic interpenetrating networks, Nature Materials Vol. 5, No. 6, pp. 494-501

Vincent, J. F. V. (2000), Smart by name, smart by nature, Smart Materials and Structures, Vol. 9, No. 3, pp. 255-259.

Watanabe, J. and Ishihara, K. (2008), Establishing ultimate biointerfaces covered with phosphorylcholine groups", Colloids and Surfaces B: Biointerfaces, Vol. 65, No. 2, pp. 155-165.

Winch, R. P., Photoelectric Properties of Thin Unbacked Gold Films, (1931). Physical Review Vol. 38, pp. 321- 324.

Yamauchi, A.; Shin, Y.; Shinozaki, M. and Kawabe, M. (2000), Membrane characteristics of composite collodion membrane: IV. Transport properties across blended collodion/Nafion membrane, Journal of Membrane Science, Vol. 170, No. 1, pp. 1-7.

Zhang, S. and Ali, N. (2007), Nanocomposite Thin Films and Coatings: Processing, Properties and Performance, Imperial College Press, London, ISBN-13: 978-1860947841.

Zhou, B; Wang, L ; Mehta, N ; Morshed, S.; Erdemir, A.; Eryilmaz, O. and Prorok, B. C. (2006), The mechanical properties of freestanding near-frictionless carbon films relevant to MEMS, Journal of Micromechics and Microengineering, Vol. 16, pp. 13741381 


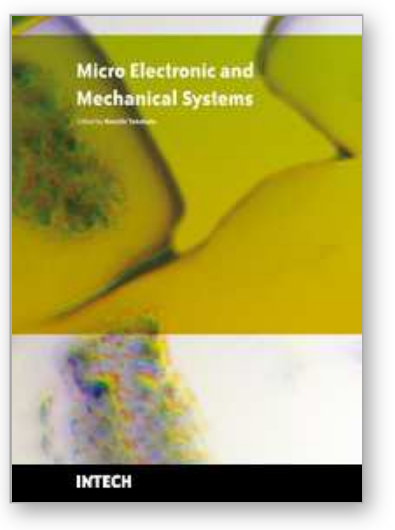

\author{
Micro Electronic and Mechanical Systems \\ Edited by Kenichi Takahata
}

ISBN 978-953-307-027-8

Hard cover, 386 pages

Publisher InTech

Published online 01, December, 2009

Published in print edition December, 2009

This book discusses key aspects of MEMS technology areas, organized in twenty-seven chapters that present the latest research developments in micro electronic and mechanical systems. The book addresses a wide range of fundamental and practical issues related to MEMS, advanced metal-oxide-semiconductor (MOS) and complementary MOS (CMOS) devices, SoC technology, integrated circuit testing and verification, and other important topics in the field. Several chapters cover state-of-the-art microfabrication techniques and materials as enabling technologies for the microsystems. Reliability issues concerning both electronic and mechanical aspects of these devices and systems are also addressed in various chapters.

\title{
How to reference
}

In order to correctly reference this scholarly work, feel free to copy and paste the following:

Jovan Matovic and Zoran Jakšić (2009). Nanomembrane: A New MEMS/NEMS Building Block, Micro Electronic and Mechanical Systems, Kenichi Takahata (Ed.), ISBN: 978-953-307-027-8, InTech, Available from: http://www.intechopen.com/books/micro-electronic-and-mechanical-systems/nanomembrane-a-newmems-nems-building-block

\section{INTECH}

open science | open minds

\section{InTech Europe}

University Campus STeP Ri Slavka Krautzeka 83/A 51000 Rijeka, Croatia Phone: +385 (51) 770447

Fax: +385 (51) 686166 www.intechopen.com

\section{InTech China}

Unit 405, Office Block, Hotel Equatorial Shanghai No.65, Yan An Road (West), Shanghai, 200040, China 中国上海市延安西路65号上海国际贵都大饭店办公楼405单元 Phone: +86-21-62489820

Fax: +86-21-62489821 
(C) 2009 The Author(s). Licensee IntechOpen. This chapter is distributed under the terms of the Creative Commons Attribution-NonCommercialShareAlike-3.0 License, which permits use, distribution and reproduction for non-commercial purposes, provided the original is properly cited and derivative works building on this content are distributed under the same license. 